\title{
Rejuvenation of the centre of the face: a new paradigm. Endoscopic lifting with fat grafting
}

\author{
Gabriel Pignata ${ }^{1}$, Alessandro Gennai ${ }^{2}$, Francesco Bernardini ${ }^{3}$ \\ 'Ophthalmology Department, British Hospital, Montevideo 11600, Uruguay. \\ ${ }^{2}$ Gennai Chirurgia, Bologna 40122, Italy. \\ ${ }^{3}$ Oculoplastica Bernardini, Casa di Cura Villa Serena, Genova 16145, Italy.
}

Correspondence to: Dr. Gabriel Pignata, Ophthalmology Department, British Hospital, Av Italia 2420, Montevideo 11300, Uruguay. E-mail: gabriel@oculoplastica.uy

How to cite this article: Pignata G, Gennai A, Bernardini F. Rejuvenation of the centre of the face: a new paradigm. Endoscopic lifting with fat grafting. Plast Aesthet Res 2018;5:23. http://dx.doi.org/10.20517/2347-9264.2018.28

Received: 20 Apr 2018 First Decision: 12 Jun 2018 Revised: 28 Jun 2018 Accepted: 29 Jun 2018 Published: 20 Jul 2018

Science Editor: Raffaele Rauso, Raúl González-García Copy Editor: Jun-Yao Li Production Editor: Huan-Liang Wu

\begin{abstract}
The ageing process starts in the centre of the face in the periocular region and around the mouth, with a combination of volume loss and tissue descent. It is a logical approach to assess all these problems at once. The combination of minimal incisions vertical endoscopic lifting combined with superficial enhanced fluid fat injection can be an integral approach to facial rejuvenation.
\end{abstract}

Keywords: Minimal incisions vertical endoscopic lifting, superficial enhanced fluid fat injection, endoscopic lifting, fat grafting

\section{INTRODUCTION}

Scientific studies have proven the central role of the eyes in aesthetic facial rejuvenation. Nguyen et al. ${ }^{[1]}$ used an eye-tracking system to demonstrate that age and fatigue judgments are related to preferential attention toward the eye region. Consequently, aesthetic surgery to the eye region may be one of the most effective interventions in enhancing the overall appearance of an individual ${ }^{[1]}$. A recent prospective study on 72 female patients with an average age of 48 years, showed that $74.6 \%$ of these patients felt that their first sign of ageing occurred in the eyes, $26.7 \%$ in the neck and only $12.6 \%$ in the cheeks ${ }^{[2]}$. Among this female group, $78.8 \%$ of patients expressed interest in improving the appearance of their eyes, $28.8 \%$ the cheeks and lips only $15 \%{ }^{[2]}$.

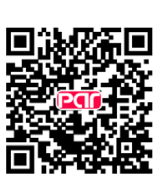


The ageing process that occurs in this age group affects the centre of the face, represented by the periocular region and the mouth. Therefore, surgeons dedicated to the periocular region should look beyond the eyelids. In a previous publication, Bernardini et al. ${ }^{[3]}$ defined the periocular aesthetic unit as being formed by the superior complex (SC), which includes the forehead, brow and upper eyelid, the inferior complex (IC), which includes the lower eyelid and the cheek, and the lateral complex (LC) which includes the temple, the malar mound and the lateral canthus. If we expand our focus to the peri-oral unit we can assess the whole centre of the face.

In the past, aesthetic surgeons dedicated their efforts to the rejuvenation of the contour of the face, offering variably satisfying results at the cost of long scar lines, extensive dissection and strong horizontal traction with unnatural effects. Vertically repositioning the descended tissue seems to be the correct way to assess the gravitational ageing vector. This is especially true in the SC and in a lesser proportion in the IC. Minimal incision vertical endoscopic lifting (MIVEL) can obtain such results with invisible scars and fast postoperative recovery. However, as much as we lift tissue, there is no natural, three-dimensional way to achieve rejuvenation without volume restoration to the deflated areas. Superficial enhanced fluid fat injection (SEFFI) is a novel technique, which not only provides the volume restoration of autologous fat, but also aids in skin regeneration.

The authors attempt to review the literature on this two novel procedures as well as present their individual techniques and results.

\section{MIVEL}

There are a few specific surgical instruments, but the single most important one is the endoscopic elevator which slides on the fiberoptic and allows the surgeon to elevate the tissue while viewing with one hand.

\section{Blind dissection}

After a Klein-type of local anesthesia and with the patient under intravenous sedation the intervention requires initial dissection in preparation for the endoscopic view. Three vertical $1.5-\mathrm{cm}$ long incisions are placed in the scalp, right behind the hairline: the central one in the midline and two paramedian incisions, one on each side, $5 \mathrm{~cm}$ from the midline, to give access to the frontal area up to the temporal crest. Two $3-\mathrm{cm}$ long incisions are performed in the temporal region, one on each side, to provide access to the temporal, malar, and zygomatic regions. In the frontal area, blind dissection is performed under the periosteum upto a 2 -cm safety line above the superior orbital rim. Special care needs to be taken in order not to elevate the conjoint tendon at this time. The lateral extension will be up to the temporal crest laterally and $2-3 \mathrm{~cm}$ posterior to the hair line. In the temporalis area an optical pocket is created blindly and the dissection plane lies above the superficial plane of the deep temporal fascia. From the temporal dissection the conjoint tendon can be safely elevated, joining both pockets.

\section{Endoscopic dissection}

After the blind dissection is completed and a single optical pocket formed in the frontal and temporal areas, it is then time to enter with the endoscope. In the temporal region, the dissection is carried out on the surface of the temporal fascia past the sentinel vein to expose the zygomatic temporal and zygomatic facial bundles laterally and the lateral retinaclum and lateral canthal tendon medially. In the forehead region, subperiostal direction is carried up to the superior orbital rim. At this level, periosteum will be elevated from lateral to medial, around the supraorbital nerve, and past it towards the glabellar region, elevating the corrugator and procures muscles.

\section{Fixation}

Fixation was achieved with Endotine devices in more than 300 cases, encountering device related complica- 
tions in almost $10 \%$ of the cases (exposure, infection, visibility, rupture of the device during implantation, trephination hemorrhage), even having to remove the device in three cases ${ }^{[3]}$. One of the authors, A.G., developed a stitch for paramedian fixation with the Reverdin needle, which will be referred to as the Gennai stitch henceforth. The Gennai stitch has successfully been used for paramedian fixation in over 200 patients without complications. Two stab wounds are performed in the forehead at a certain distance from the paramedian incision. The Reverdin needle is passed though the stab incision and exits at the paramedian incision, and the suture is loaded and brought back to the stab incision, where only one of the branches is brought back with the Reverdin needle through the subcutaneous tissue back to the paramedian incision, where it is freed and tied to the other branch, thus grasping the tissue and elevating the eyebrow. This stitch will cause a characteristic bulging that will disappear in the postoperative period.

Temporal fixation of the deep subcutaneous tissue of the elevated flap to the deep temporal fascia is simply performed with a 3/0 Vicryl suture. Due to the extensive dissection, the inferior complex and the lateral complex will be elevated as a whole with this fixation.

Bicoronal fixation can be used in 50\% of the cases to enhance the elevation of the eyebrows.

\section{SEFFI}

\section{Harvesting}

For the preparation of the tissue for harvesting a solution of lidocaine $(400 \mathrm{mg})$, sodium bicarbonate $(5 \mathrm{mEq})$ and epinephrine $(1 \mathrm{mg})$, is prepared in a cold Ringer's lactate solution $(500 \mathrm{~mL})$ and it is inflitrated into the autologous collection site(s); most frequently the supra pubic region (55\%), hips (22\%), prethrocanteric area $(18 \%)$, inner thigh $(3 \%)$, and inner knee $(2 \%)^{[4]}$. For SEFFI, manual aspiration of fat is performed with the use of a $20 \mathrm{~cm}$-long multi perforated cannula (with other $0.5-\mathrm{mm}$ or $0.8-\mathrm{mm}$ ports) mounted in a $10-\mathrm{mL}$ syringe; for micro-SEFFI, with a $15-\mathrm{cm}$ long multi perforated cannula with $0.3-\mathrm{mm}$ ports. Manual aspiration is begun 15 min after infiltration of the preparation solution.

The aspirated material ( $0.6 \mathrm{~mL}$ per syringe) is cleaned with Ringer's solution $(0.4 \mathrm{~mL})$ and left to decant in vertical position for about $2 \mathrm{~min}$. The liquid part collected at the bottom of the syringe is discarded. This procedure is repeated once again to ensure that most of the blood and the anesthetic solution is eliminated from the fat ${ }^{[5]}$. The syringes are kept capped under a sterile cloth.

Autologous blood is drawn from the patient to obtain platelet rich plasma (PRP). It is poored into $4.5 \mathrm{~mL}$ citrated Vacutainer and centrifuged at $2000 \mathrm{rpm}$ for $4 \mathrm{~min}$. The fat and PRP are mixed so that PRP is $20 \%$ of the total harvested tissue ${ }^{[6]}$.

\section{Injection}

The solution is then injected according to a comprehensive study of the facial arterial system, using a linear retrograde injection technique. The M-SEFFI technique compliments the SEFFI technique; SEFFI performed with cannula with distal ports of $0.8 \mathrm{~mm}$ is indicated for the larger volume defects in the cheek, temple, forehead, chin and jaw line, and SEFFI performed with cannula with distal ports of $0.5 \mathrm{~mm}$ is indicated for brow and lip volume restoration. M-SEFFI is best for the upper sulcus and infra orbital hollows and the fine wrinkles in the periocular area, such as the smile lines that form in the lower eyelid, and the perioral fine lines of the lips ${ }^{[4]}$.

\section{DISCUSSION}

In the past, surgeons have focused on the rejuvenation of the frame of the face through extensive facelifts and the periocular structures individually, correcting brow ptosis, dermatochalasis, eyelid bags, tear trough 


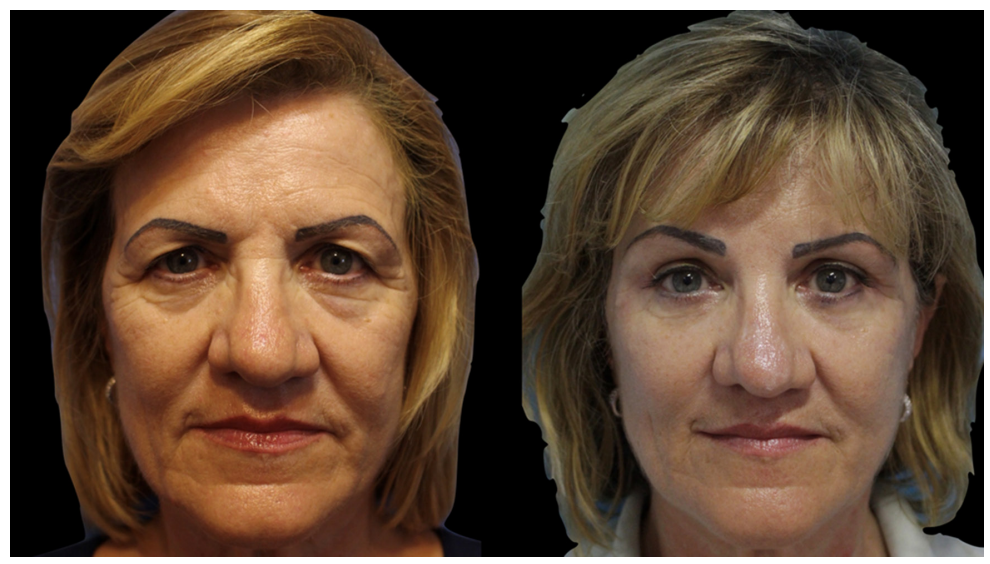

Figure 1. Pre-treatment and 6 months post-treatment views of a 53-year-old woman treated with MIVEL and SEFFI in her cheeks, periocular and perioral regions combined with four-lid blepharoplasty

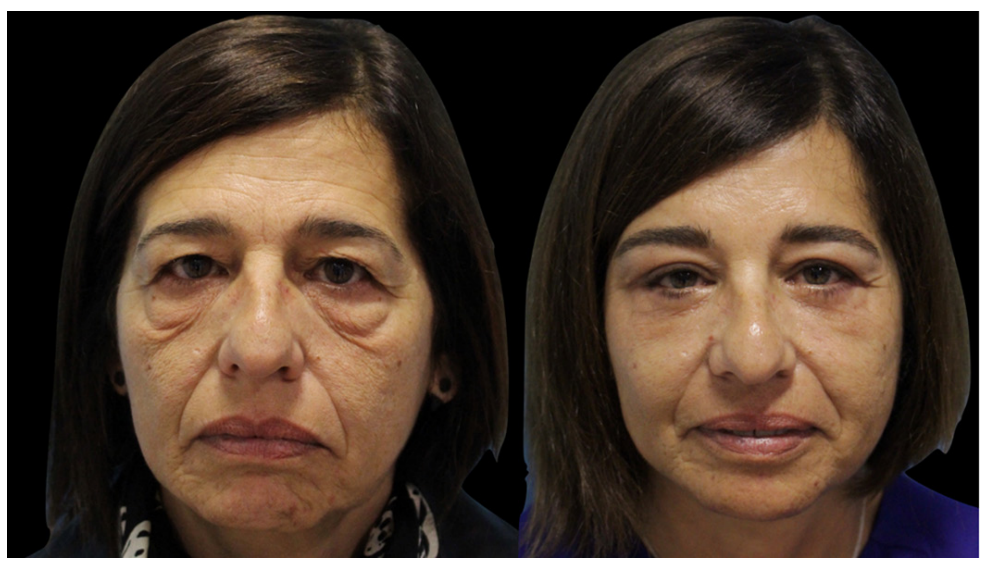

Figure 2. Pre-treatment and 6 months post-treatment views of a 51-year-old woman treated with MIVEL and SEFFI in her cheeks, periocular and perioral regions combined with four-lid blepharoplasty

deformities and fat pads descent or atrophy in variable combinations without a systematical approach. The knowledge of the events taking place in the ageing process has evolved and the authors believe that a paradigm change in the approach of the ageing of the periocular region as a single aesthetic unit is in order. Liew and Nguyen ${ }^{[7]}$ recognised two types of patients, those showing signs of tissue descent and those showing signs of volume depletion. The authors believe that, as surgeons, we should consider this two aspects of ageing as both present in a certain degree and analyze the effect of both in the different complexes of the periocular region in the preoperative evaluation of our patients, in order to offer a systematic and global solution to our patients demands. In the periocular unit, the SC is affected by both descent and deflation, the IC is affected by deflation and descent in a leer degree and the LC is predominantly affected by deflation. A systematic approach to the aesthetic unit carries a high satisfaction rate ${ }^{[8,9]}$. MIVEL is a scarless technique that assesses the vertical vector of tissue descent that occurs in the SC. However, for as much as tissue can be lifted, a 3-dimensional rejuvenation of the periocular esthetic unit can be only achieved if associated with volume restoration ${ }^{[10,11]}$. SEFFI and M-SEFFI allow for the correction of volume depletion in the finer and more superficial areas closer to the eyes and lips as well as the deeper areas of the forehead, eyebrows, temples and cheeks, assessing not only volume depletion, but also adding skin rejuvenation due to its content of adiposederived stem cells, with a lower rate of complications compared to traditional fat grafting techniques ${ }^{[12-14]}$. The authors believe that the main advantage of these techniques used in combination is that they assess the mayor consequences of aging in one integral surgical act, thus achieving more natural results than the previous horizontal tightening procedures. 
Skin removal and fat removal-repositioning techniques can be effectively associated to these procedures when indicated. Clinical examples in Figures 1 and 2.

\section{CONCLUSIONS}

A systematic approach to aesthetic units carries a high satisfaction rate. With the goal of rejuvenating the periocular region, the authors advocate approaching this area as a unit rather than with individual isolated techniques that can achieve only limited improvements. MIVEL is a scarless technique that respects the vertical vector of tissue descent. As much as tissue can be lifted, a 3-dimensional rejuvenation of the periocular aesthetic unit can only be achieved if associated with volume restoration. SEFFI and M-SEFFI techniques assess this issue with a high satisfaction and a low complication rate.

\section{DECLARATIONS}

\section{Authors' contributions}

Assistant surgeon in part of the cases, research and writing,: Pignata G

Surgeon in charge of part of the cases: Gennai A

Original idea, surgeon in charge of part of the cases, and processing pictures: Bernardini $\mathrm{F}$

\section{Availability of data and materials}

The data were strictly obtained from medical records according to the privacy policy and ethics code of our institute.

\section{Financial support and sponsorship}

None.

\section{Conflicts of interest}

All authors declared that there are no conflicts of interest.

\section{Ethical approval and consent to participate}

Informed consent was obtained for each procedure and the review adhered to the tenets of the Declaration of Helsinki.

\section{Consent for publication}

Informed consent was obtained for publication.

\section{Copyright}

(c) The Author(s) 2018.

\section{REFERENCES}

1. Nguyen HT, Isaacowitz DM, Rubin PA. Age- and fatigue-related markers of human faces: an eye-tracking study. Ophthalmology 2009;116:355-60.

2. Sobanko JF, Taglienti AJ, Wilson AJ, Sarwer DB, Margolis DJ, Dai J, Percec I. Motivations for seeking minimally invasive cosmetic procedures in an academic outpatient setting. Aesthet Surg J 2015;35:1014-20.

3. Bernardini FP, Gennai A, Izzo L, Devoto MH. Minimal incisions vertical endoscopic lifting and fat grafting as a systematic approach to the rejuvenation of the periocular esthetic unit. Ophthal Plast Reconstr Surg 2013;29:308-15.

4. Gennai A, Zambelli, A, Repaci E, Quarto R, Baldelli I, Fraternali G, Bernardini FP. Skin rejuvenateion and volume enhancement with the micro superficial enhanced fluid fat injection (M-SEFFI) for skin aging of the periocular and perioral region. Aesthet Surg J 2017;37:1423.

5. Girard AC, Atlan M, Bencharif K, Gunasekaran MK, Delarue P, Hulard O, Lefebvre-d'Hellencourt C, Roche R, Hoareau L, Festy F. New 
insights into lidocaine and adrenaline effects on human adipose stem cells. Aesthetic Plast Surg 2013;37:144-52.

6. Li F, Guo W, Li K, Yu M, Tang W, Wang H, Tian W. Improved fat graft survival by different volume fractions of platelet-rich plasma and adipose-derived stem cells. Aesthet Surg J 2015;35:319-33.

7. Liew S, Nguyen DQ. Nonsurgical volumetric upper periorbital rejuvenation: a plastic surgeon's perspective. Aesthetic Plast Surg 2011;35:319-25.

8. Ramirez OM. The central oval of the face: tridimensional endoscopic rejuvenation. Facial Plast Surg 2000;16:283-98.

9. Citarella ER, Sterodimas A, Conde-Green A. Endoscopically assisted limited incision rhytidextomy: a 10-year prospective study. J Plast Reconstr Aesthet Surg 2010;63:1842-8.

10. Kranendonk S, Obagi S. Autologous fat transfer for periorbital rejuvenation: indications, technique, and complications. Dermatol Surg 2007;33:572-8.

11. Glasgold M, Lam SM, Glasgold R. Volumetric rejuvenation of the periorbital region. Facial Plast Surg 2010;26:252-9.

12. Derby BM, Dai H, Reichensperger J, Cox L, Harrison C, Cosenza N, Yang M, Bueno RA, Neumeister MW. Adipose-derived stem cell to epithelial stem cell transdifferentiation: a mechanism to potentially improve understanding of fat grafting's impact on skin rejuvenation. Aesthet Surg J 2014;34:142-53.

13. Al-Nbaheen M, Vishnubalaji R, Ali D, Bouslimi A, Al-Jassir F, Megges M, Prigione A, Adjaye J, Kassem M, Aldahmash A. Human stromal (mesenchymal) stem cells from bone marrow, adipose tissue and skin exhibit differences in molecular phenotype and differentiation potential. Stem Cell Rev 2013;9:32-43.

14. Bernardini FP, Gennai A, Izzo L, Zambelli A, Repaci E, Baldelli I, Fraternali-Orcioni G, Hartstein ME, Santi PL, Quarto R. Superficial enhanced fluid fat injection (SEFFI) to correct volume defects and skin aging of the face and periocular region. Aesthet Surg J 2015;35:50415. 